\title{
8. Schlussfolgerungen und Ausblick
}

Diese Arbeit hat den barrierefreien bzw. -reduzierten Wohnraum als Gegenstand politischer Steuerung untersucht. Dafür wurden die Bundesländer als zentrale föderale Ebene in Deutschland identifiziert. Erstens interessierte, in welchen Ausprägungen die 16 Länder die ihnen zur Verfügung stehenden politischen Maßnahmen nutzen: insbesondere bauordnungsrechtliche Vorgaben zur Barrierefreiheit, die soziale Wohnraumförderung und Wohnberatungen zur Wohnraumanpassung. Zweitens sollte darauf basierend herausgefunden werden, was die zu diesen Maßnahmen führenden politischen Prozesse in einzelnen Bundesländern kennzeichnet. Dafür stellte der Ansatz eigendynamischer politischer Prozesse (AEP) eine politikfeldanalytische Heuristik dar, die die Fallstudien zu Sachsen-Anhalt und Hessen theoretisch gerahmt hat.

Das Ziel war es, einerseits theorieorientierte neue politikwissenschaftlichen Erkenntnisse zu einem bislang so gut wie nicht beachteten Forschungsthema zu generieren und andererseits ein gesellschaftspolitisch relevantes Thema zu bearbeiten. Die folgenden Abschnitte beschäftigen sich mit diesen beiden Ansprüchen. Nach einer zusammenfassenden Diskussion der zentralen Erkenntnisse erfolgt eine Reflexion zum Ansatz eigendynamischer politischer Prozesse. Dabei sollen die eigenen Ergebnisse auch in breitere politikwissenschaftliche Debatten eingeordnet werden. Dann wird das eigene methodische Vorgehen kritisch hinterfragt, um mögliche Schwachstellen sowie weitere Forschungsdesiderata $\mathrm{zu}$ identifizieren. Abschließend sind verschiedene wohnungs- und sozialpolitische Implikationen zu diskutieren, die im Rahmen der Analyse sichtbar geworden sind.

Zusammenfassung der zentralen Erkenntnisse

Die erste Forschungsfrage war, in welchen Ausprägungen die Bundesländer politische Maßnahmen (»Policies«) zur Steuerung von barrierefreiem bzw. -reduziertem Wohnraum nutzen. Hier hat die empirische Analyse gezeigt, dass dieser wohnungspolitische Teilbereich von den 16 Bundesländern unterschiedlich stark und in unterschiedlicher Weise gesteuert wird (vgl. Kapitel 6). Insgesamt sind Bayern, Niedersachen, NordrheinWestfalen sowie Rheinland-Pfalz besonders aktiv-steuernde Bundesländer. Anders als erwartet zeigt sich keine Tendenz dahingehend, dass einige Länder stärker regulativ und andere Länder über weichere Formen der ökonomischen Anreizsteuerung oder 
Information den barrierefreien bzw. -reduzierten Wohnraum adressieren. Vielmehr hat ein Bundesland diese Thematik entweder für sich erkannt oder aber widmet die politische Aufmerksamkeit anderen wohnungs- bzw. sozialpolitischen Themen. Gleichzeitig unterliegt das Feld einer Dynamik, die auch das Verhältnis der einzelnen Länder untereinander verändern kann. Bremen etwa verschärft sein Bauordnungsrecht zum Jahr 2021 und Berlin hat zum Januar 2020 als erstes Bundesland eine eigene Verordnung spezifisch zum barrierefreien Bauen erlassen.

Die zweite Forschungsfrage zielte darauf ab, das Zustandekommen bzw. Ausbleiben sowie die konkrete Ausgestaltung dieser Policies zu erklären. Sachsen-Anhalt und Hessen gelten nach den vorherigen Erkenntnissen nicht als aktiv-steuernde Bundesländer, aber dennoch verlaufen die politischen Prozesse in beiden Bundesländern sehr unterschiedlich (vgl. Kapitel 7). Auf der Suche nach Erklärungen für diese Varianz erwies sich der AEP als eine zweckmäßige Heuristik. Die fünf AEP-Erklärungsfaktoren besitzen in unterschiedlicher Stärke und teils mit landesspezifischen Besonderheiten Erklärungskraft. So beeinflussen die Problemstrukturen - insbesondere der mutmaßliche Zielkonflikt zwischen einerseits bezahlbarem und andererseits barrierefreiem Wohnraum sowie die Situation auf den regionalen Wohnungsmärkten - maßgeblich das Handeln der politischen Entscheidungsträger*innen $(\rightarrow$ Zielkonflikte, sozioökonomische Schule). Die Problemstrukturen selbst sind wiederum erst ein Ergebnis von interessengeleiteten Interpretationen der am politischen Prozess beteiligten Akteure. Hier zeigt sich ein inkrementeller, langfristiger Politikstil, der dem Bild einer auf spontane Stimmungswechsel in der Bevölkerung ausgerichteten Politik widerspricht und sich besonders stark in Low-LevelSettings mit geringer politischer und öffentlicher Aufmerksamkeit manifestiert. Selbiges gilt für die genutzten Instrumente und die diskutierten Instrumenten-Alternativen $(\rightarrow$ Policy-Mix). Vielfach fordern Interessengruppen jahrelang einen Politikwandel ein, bis sich genügend Unterstützer*innen für seine Durchsetzung finden und bestehende institutionelle sowie ideologische Filter überwunden werden ( $\rightarrow$ Interessengruppen). An dieser Stelle können situative Aspekte als Möglichkeits- oder als Unmöglichkeitsfenster wirken, um eine Instrumenten-Alternative zu unterstützen oder zu hemmen. Hier wird die begrenzt rationale Eigendynamik politischer Prozesse deutlich, die nicht immer an der Dringlichkeit eines politischen Problems ausgerichtet sein müssen $\rightarrow$ begrenzte Rationalität). Vielmehr entscheidet sich erst im Zusammenspiel der jeweils beteiligten Akteure, welche Instrumenten-Alternative sich nicht nur im Hinblick auf ihre technische und finanzielle Machbarkeit, sondern auch im Hinblick auf ihre politische Durchsetzbarkeit umsetzen lässt. Als »Satisficer« wählen die Akteure dabei auf Basis der ihnen vorhandenen Kenntnisse eine »ausreichend gute« Lösung zur Adressierung des identifizierten politischen Problems (vgl. Simon 1972: 168). Die formal rein ausführende Ministerialverwaltung verfügt dabei über ein großes Potential zur strategischen Interaktion, während die Parteipolitik sich angesichts ihrer begrenzten Ressourcen vielfach auf die großen politischen Linien beschränken muss ( $\rightarrow$ Verwaltungswissenschaft und Policyanalyse). Neben der formalen Zuschreibung bestimmen allerdings immer auch die ein jeweiliges Amt bekleidenden Individuen mit ihren eigenen Wertvorstellungen und Netzwerken, wie sehr sie sich für oder gegen ein bestimmtes politisches Vorhaben einsetzen ( $\rightarrow$ Policy Entrepreneure). Aus institutioneller Perspektive kann außerdem die sektorale Politikintegration einer an Problemlösung orientierten Politik im Wege stehen. 
Zwar finden sich sowohl in Sachsen-Anhalt als auch in Hessen Versuche des Disability Mainstreaming, um inklusionspolitische Belange stärker in die Wohnungspolitik zu integrieren. Diese stoßen allerdings in der praktischen Umsetzung an ihre Grenzen und können nur bedingt als erfolgreich gelten $(\rightarrow$ Politikintegration). Gerade das Zusammenwirken der einzelnen AEP-Erklärungsfaktoren vermag es somit, komplexe politische Prozesse zu erklären. Diese zentrale Grundannahme aus dem AEP bestätigt sich auch beim barrierefreien bzw. -reduzierten Wohnraum (vgl. Böcher/Töller 2019: 197f.).

Die retrospektive Überprüfung von Sachsen-Anhalt und Hessen als prototypische Fallstudien lässt folgende Schlussfolgerungen zu (vgl. Kapitel 5.2.1). Sachsen-Anhalt erscheint als Prototyp für ein demografisch alterndes Bundesland mit entspannten Wohnungsmärkten. Dieser sozioökonomische Kontext prägt die Politikgestaltung zum barrierefreien bzw. -reduzierten Wohnraum und ermöglicht eher konsensgeprägte Politikprozesse, in denen die Angebots- und Nachfrageseite nach Wohnraum an einer gemeinsamen Problemlösung interessiert sind. In Hessen wiederum konzentriert sich der wohnungspolitische Diskurs auf die Wohnraumknappheit im Rhein-Main-Gebiet, womit qualitative Zusatzbedarfe wie der barrierefreie bzw. -reduzierte Wohnraum von der Agenda gespült werden. Auch diese Erkenntnis widerspricht einer rationalen Problemlösung insofern, als dass die hessischen Wohnungsmärkte in sich heterogen sind und einer stärker differenzierten politischen Steuerung bedürften. Davon abgesehen wäre anzunehmen, dass sich in anderen Bundesländern mit ähnlich angespannten Wohnungsmärkten ähnliche Policy Outputs finden. Die Typologie politischer Maßnahmen zeigt jedoch umgekehrt, dass die Stadtstaaten Bremen und Berlin die Herstellung von barrierefreiem Wohnraum in ihrem Bauordnungsrecht zunehmend verstärkt steuern. Auch lässt der Erklärungsversuch über demografische Strukturen und die Lage auf den Wohnungsmärkten keine befriedigende Erklärung für die aktive Steuerung in Bayern, Niedersachen, Nordrhein-Westfalen und Rheinland-Pfalz zu. Folglich bedarf es zu einer Validierung und Erweiterung der hier getroffenen Annahmen weiterer Forschung: Inwieweit spielen parteipolitische Faktoren eine Rolle und welches Gewicht kommt der Professionalisierung und Artikulationskraft von Veränderungsinteressen für eine stärkere Barrierefreiheit in der Wohnungspolitik zu? Wie ist zu erklären, dass neben den herausgearbeiteten aktiv-steuernden Beispielen der Großteil der Bundesländer bislang eher verhalten in diesem wohnungspolitischen Teilbereich agiert?

\section{Diskussion zum AEP und Einordnung in breitere politikwissenschaftliche Debatten}

Bislang wurde der AEP vor allem auf umweltpolitische Fragestellungen angewandt (vgl. etwa Töller/Böcher 2016; Töller/Böcher 2017; Jara 2020). In der vorliegenden Arbeit hat er allerdings eine sehr gute Anwendbarkeit auch über die Umweltpolitikforschung hinaus bewiesen. Die Fallstudien ließen sich durch die fünf Erklärungsfaktoren strukturieren und dem Erkenntnisinteresse entsprechend durch weitere theoretische Annahmen verfeinern. Im Folgenden sollen Herausforderungen im Umgang mit der Forschungsheuristik erörtert sowie Potentiale zu ihrer weiteren Entwicklung aufgezeigt werden.

Herausfordernd bei Heuristiken generell ist der Spagat zwischen dem Allgemeinen und dem Konkreten. Sind die Annahmen zu allgemein gehalten, entstehen nicht falsifizierbare Allgemeinplätze und die Erklärungskraft wird geschmälert. Je konkreter 
hingegen die Annahmen, desto schwieriger die Anwendung auf unterschiedliche Fragestellungen (vgl. Wenzelburger/Zohlnhöfer 2015: 27f.; Reiter/Töller 2014: 27; Holtkamp 2012: 100). Dieser Spagat findet sich auch bei der Arbeit mit dem AEP, insbesondere in der konzeptionellen Ausarbeitung über seine Grundannahmen hinaus. Hier war es notwendig, vor allem die Erklärungsfaktoren »Institutionen« sowie »Akteure und ihre Handlungen« mit Blick auf die politischen Prozesse zum barrierefreien bzw. -reduzierten Wohnraum durch zusätzliche Literatur anzureichern (etwa zum föderalen Mehrebenensystem, zur Verwaltungswissenschaft und der Interessengruppenforschung). Versteht man eine Heuristik als einen solchen Rahmen, der mit je spezifischen, auf den Erkenntnisgegenstand zugeschnittenen Annahmen "gefüllt« wird, ergeben sich vielfältige theoretische Anknüpfungspunkte. Die Annahmen zur Eigendynamik, zur Wechselwirkung zwischen den Erklärungsfaktoren und zur begrenzt rationalen Politikgestaltung bleiben trotz dieser forschungsindividuellen Modifikationen bestehen und eine Vergleichbarkeit unterschiedlicher Studien auf AEP-Basis ist nach wie vor gegeben.

Die Wechselwirkungen zwischen den Erklärungsfaktoren werden der politischen Realität gerecht, stellen Forschungsvorhaben aber zugleich vor konzeptionelle Schwierigkeiten. Wenn gerade das Zusammenspiel unterschiedlicher Faktoren einen politischen Prozess erklärt, kann darunter die analytische Trennschärfe zwischen den einzelnen Erklärungsfaktoren sowie ihre individuelle Wirksamkeit leiden. So sind institutionelle Regelungen und Akteure durch ihre gegenseitige Abhängigkeit eng miteinander verwoben. Und auch die Problemstrukturen waren in ihrer empirischen Anwendung eine Herausforderung, da sie einerseits »dem Untersuchungsgegenstand inhärent « sind, andererseits aber maßgeblich durch sozioökonomische, situative und akteursspezifische Aspekte beeinflusst werden. Die sozioökonomische Schule begreift »Staatstätigkeit vor allem als Reaktion auf gesellschaftliche und wirtschaftliche Entwicklungen « (Schmidt/Ostheim 2007b: 29) und ist nicht dezidiert im AEP integriert, sodass sich die Frage stellt, wo und inwieweit derartige langfristige Entwicklungen ihren Raum finden. Als externe Faktoren könnten sie den situativen Aspekten zugeordnet werden. Diese wurden in der vorliegenden Arbeit aber als plötzliche, weitgehend unerwartete Gelegenheitsfenster verstanden. Bereits die Namensgebung zu »situativen « Aspekten unterstreicht den kurzfristigen, spontanen Charakter dieses Erklärungsfaktors. Langfristige Wirkungen zur demografischen Entwicklung und damit verbundenen veränderten Wohnraumbedarfen, aber auch zur Haushaltslage der Länder oder zur Ausbreitung des Inklusionsgedankens, fallen nicht unter ein solches Verständnis. Gleichzeitig sind sie auch nicht dem Untersuchungsgegenstand selbst inhärent, sodass eine Verortung unter den Problemstrukturen ebenfalls zweifelhaft erscheint. Diese mangelnde Berücksichtigung sozioökonomischer, inkrementeller Faktoren teilt sich der AEP mit dem AZI und dem MSA (vgl. Schmidt/Ostheim 2007b: 34f.; Obinger 2015: 49f.). Vielfach wird schlicht vorausgesetzt, dass solche Entwicklungen politische Prozesse beeinflussen. Wie für den barrierefreien bzw. -reduzierten Wohnraum gezeigt worden ist, ist dieser Einfluss allerdings nicht zu unterschätzen und verdient einen Platz in politikfeldanalytischen Forschungsheuristiken.

Ferner wurden die »Instrumente und Instrumenten-Alternativen« stärker theoretisch ausgearbeitet. Inwieweit handelt es sich um wahrliche Instrumenten-Alternativen, nach denen etwa ein regulatives anstelle eines ökonomischen Instruments angewandt 
wird, oder um kleinteiligere Alternativen wie den Ersatz einer ökonomischen Maßnahme durch eine andere oder lediglich Veränderungen innerhalb einer bestehenden Maßnahme? Um dieses Spektrum konzeptionell abbilden zu können, hat die vorliegende Arbeit die Instrumenten-Alternativen nach Typ 1 bis 3 entwickelt. Hieraus folgt die Erkenntnis, dass bereits »kleinere« Politikwandel im Rahmen einer bestehenden Policy intensive politische Debatten und weitreichende Policy-Wirkungen mit sich bringen können. Solche Instrumenten-Alternativen nach Typ 1 machen den Großteil des politischen Alltagsgeschäfts aus. Auch wenn die Politikfeldanalyse ihre Aufmerksamkeit gern gänzlich neuen Maßnahmen schenkt - etwa dem Kohleausstieg oder einer CO2-Abgabe -, erscheint es deshalb lohnend, auch die inkrementellen Entwicklungen in bestehenden Maßnahmen wissenschaftlich zu begleiten.

Trotz der hier benannten Herausforderungen in der Arbeit mit dem AEP hat er sich als sehr überzeugendes Analyseinstrument erwiesen. Durch seine Grundannahmen bietet er eine theoretische Handlungsanleitung, die in der Lage ist, forschungsspezifische Modifikationen zuzulassen. Das zeigte sich am deutlichsten ebenfalls bei den "Instrumenten und Instrumenten-Alternativen«, da die vorliegende Forschungsarbeit keine einzelne Policy-Entscheidung, sondern einen Policy-Mix zum Erklärungsgegenstand hatte. Dieses politikfeldanalytisch eher unübliche Vorgehen ließ sich problemlos in die AEP-Heuristik integrieren und führte zu vielversprechenden Anknüpfungspunkten für weitere Forschungsvorhaben. So ließen sich die Erkenntnisse zu Zielkonflikten, zur Politikintegration und zur identifizierten breiten, an den politischen Prozessen beteiligten Akteurslandschaft erst durch das Zusammenspiel der einzelnen Policies angemessen erfassen. Zur Lösung der meisten politischen Probleme ist eine Kombination von politischen Maßnahmen notwendig (vgl. Kapitel 4.2.1), womit die analytische Beschränkung auf eine einzelne Policy zwangsweise blinde Flecken erzeugt und dazu verleitet, Zielkonflikte zu übersehen bzw. nur aus einer einzelnen Perspektive zu beleuchten. Gerade weil ein Policy-Mix häufig die üblichen Ressort- und Politikfeldgrenzen außer Kraft setzt, erscheint die Untersuchung derart interdependenter Maßnahmenbündel reizvoll (zur Trennschärfe von Politikfeldern vgl. Döhler 2015, Haunss/Hofmann 2015 und Loer et al. 2015). In diesem Atemzug könnte die Literatur zum Policy-Mix stärker in die Phase der Politikformulierung integriert werden: Inwieweit beeinflussen Zielsetzungen zur kohärenten, policy-übergreifenden Politikgestaltung die politischen Prozesse selbst? Im Sinne einer rationalen, an Problemlösung orientierten Politikgestaltung sollten einzelne Maßnahmen aufeinander abgestimmt und bei Bedarf neu angepasst werden. Dieser idealtypische Ablauf kann durch unterschiedliche Ressortlogiken und widerstreitende Interessen jedoch geschmälert werden. Wie Böcher/Töller (2012: 186f.) unterstellen, muss nicht immer die Problemlösung die zentrale Motivation in politischen Prozessen sein - die Folge kann ein inkohärenter Policy-Mix sein, der in der Literatur unter »drift«, »conversion« oder »layering« diskutiert wird (vgl. Howlett et al. 2015: 299; Rogge/Reichardt 2016: 1628; Kivimaa/Kern 2016: 206f.).

Jeder einzelne AEP-Erklärungsfaktor eröffnet durch die empirischen Erkenntnisse, die mit seiner Hilfe generiert worden sind, Ansatzpunkte zu weiteren theoretischen Debatten. Aus akteurstheoretischer Perspektive ist etwa der Mehrwert einer Verknüpfung von verwaltungswisssenschaftlichen und politikfeldanalytischen Ansätzen auszuarbeiten (vgl. Döhler 2014; Töller 2018). Aus institutioneller Perspektive zeigt das Disability 
Mainstreaming als eine spezifische Form der Politikintegration, wann inklusionspolitische Belange (keinen) Einzug in wohnungspolitische Politikentscheidungen finden. Hier besteht wiederum Anknüpfungspotential an verschiedene weitere Politikfelder, etwa zum Disability Mainstreaming im öffentlichen Raum, in der Bildungs- und Arbeitsmarktpolitik oder der Entwicklungspolitik (vgl. Grüber et al. 2011; Marshall 2012; Wacker 2013). Wenn der Blick indessen auf die breiteren wohnungspolitischen Debatten gelenkt wird, ist zu prüfen, inwieweit sich andere politikfeldferne Interessen einen Stellenwert in der Wohnungspolitik verschaffen können. Insbesondere umwelt- und klimapolitische Belange zur Energieeffizienz und erneuerbaren Energien im Gebäudesektor treten in den Interviews immer wieder als Referenz dafür auf, dass eine Politikintegration hier erfolgreicher verläuft als beim Disability Mainstreaming. In vergleichend angelegten Folgestudien ließe sich diese Annahme prüfen und untersuchen, was die Integration unterschiedlicher Belange in »fremde« Politikfelder erleichtert oder hemmt.

\section{Kritische Reflexion des eigenen Vorgehens}

Während sich die bisherigen Ausführungen auf die theoretische Rückbindung konzentrierten, geht es im Folgenden um das methodische und empirische Vorgehen der Dissertation. Die methodische Vorarbeit hatte die Gütekriterien qualitativer Sozialforschung als handlungsleitend für mein Forschungsvorhaben festgelegt (vgl. Kapitel 5.3). Insbesondere Transparenz und die intersubjektive Nachvollziehbarkeit waren wichtige Begleiter*innen über den gesamten Forschungsprozess hinweg. Nichtsdestotrotz stoßen wohl wenige Forschungsarbeiten nicht auf methodische oder empirische Herausforderungen, weshalb diese für die eigene Forschung im Folgenden reflektiert werden, um die Stärken und Schwächen der genutzten Methoden transparent darzulegen.

Der erste Teil der empirischen Analyse verglich basierend auf dem generierten empirischen Material den Policy-Mix zum barrierefreien bzw. -reduzierten Wohnraum in den 16 Ländern. Durch telefonische Interviews mit den Landesförderbanken und regionalen Ansprechpartner*innen aus der Wohnberatung wurden die vorab recherchierten Policy-Ausprägungen validiert, korrigiert oder ergänzt. Die Kleinteiligkeit und Relevanz von Detailregelungen in den einzelnen Maßnahmen erschwerten dabei eine übergeordnete vergleichende Betrachtung. Während ein tiefergehendes Verständnis des PolicyMix und seiner Querwirkungen in einem Bundesland zunächst nur ansatzweise herausgearbeitet werden konnte, bestand ein Mehrwert dieser vergleichenden Typologie darin, das bislang bestehende Spektrum politischer Steuerung in diesem politikwissenschaftlich vernachlässigten Feld überhaupt einmal zu identifizieren.

Den Anspruch zum tiefergehenden Verständnis löste der zweite Teil der empirischen Analyse für die untersuchten politischen Prozesse in Sachsen-Anhalt und Hessen ein. Hier zeigte sich, wie eng verwoben die Policies mit den im jeweiligen Land bestehenden Akteurskonstellationen, den institutionellen Rahmenbedingungen, aber auch den Problemstrukturen und unerwarteten situativen Aspekten sind. Eine Herausforderung lag dabei in der empirischen Analyse des Akteurshandelns. Die Relevanz von Akteuren für den Ausgang politischer Prozesse war offensichtlich, allerdings lassen sich Interessen, Ressourcen und Strategien nur bedingt empirisch greifen. Vor allem Formen des informellen Lobbyings oder sogenannte »hidden agendas« sind schwer 
nachzuweisen (vgl. Schiffers 2016: 481ff.; Kleinfeld et al. 2007: 13; Baruth/Schnapp 2015: 257; Reutter 2012b: 12). Während die außerparlamentarischen und parlamentarischen Interessen durch eine diversifizierte Datenbasis angemessen erfasst worden sind, stellt die Ministerialverwaltung auch nach der Analyse in Teilen noch eine »Black Box« dar. Als formal ausführende Akteurin orientiert sie sich an den politischen Leitlinien, die eigene wert- oder zweckrationale Interessen per Funktion untersagt. Entsprechend zurückhaltend waren die ministeriellen Interviewpartner*innen bei Fragen, die über ihre formale Funktion hinausgingen. An dieser Stelle verhalf eine breite Datenbasis aus parlamentarischen Dokumenten, ministeriellen Veröffentlichungen und Einschätzungen der weiteren Interviewpartner*innen zu einem »möglichst guten« Verständnis.

Generell war die Datenerhebung über leitfadengestützte Interviews eine methodische Herausforderung. So sind die am politischen Prozess beteiligten Akteure zwar vielfach Expert*innen in ihrem Feld, verfolgen zugleich aber immer eigene Interessen. Hier galt es, das jeweils generierte Wissen in einen Kontext zu setzen und im Hinblick auf die Subjektivität der Interviewpartner*innen zu filtern (Helfferich 2019: 683f.). Beispielsweise war die Nachfrageseite nach Wohnraum in Form von Behindertenvertretungen deutlich zugänglicher als die Angebotsseite in Form der Wohnungsund Bauwirtschaft. In diesem Kontext ist auch die jeweilige Funktion der Interviewpartner*innen zu reflektieren: Abhängig davon, ob es sich um die Führungs- oder die Arbeitsebene handelt, wird unterschiedliches Wissen generiert. Offensichtlich wurde die Relevanz der konkreten Funktionen auch bei den Landtagsfraktionen: Sozialpolitische Sprecher*innen vertraten naturgemäß eine andere Perspektive als ihre wohnungsbaupolitischen Kolleg*innen. Hier entschieden vielfach die Fraktionen selbst, wer als geeignete* $r$ Ansprechpartner*in zum barrierefreien bzw. -reduzierten Wohnraum galt. In der Datenauswertung wurden diese Aspekte durch eine Unterteilung in thematische Codes, Faktencodes und bewertende Codes berücksichtigt (vgl. Kapitel 5.3).

Die Akteursanalyse sollte die vielschichtigen und verflochtenen Akteursorientierungen und -beziehungen im Feld des barrierefreien bzw. -reduzierten Wohnraums modellieren, wofür korporative Akteure zunächst als homogene Einheiten skizziert worden sind (von Prittwitz 2007: 102; Schneider 2003: 110f.). Hier lässt sich argumentieren, dass es zwar immer zu Abweichungen zwischen individuellen Interessen und Partei- oder Verbandsinteressen kommen kann, aber grundsätzlich doch eine Übereinstimmung zu unterstellen ist (Holtkamp 2012: 53, 108ff.). Generell sind politische Prozesse nie vollends in ihrer Komplexität zu fassen, aber immerhin eine Annäherung sollte versucht werden. Dabei kann der »Kontrollverlust durch Komplexität< [...] als eine Art interne Fehlergröße konzipiert werden, die abhängig von der Größe, Heterogenität und Dynamik der Akteurkonstellation ist (Schneider 2003: 119). Beispielsweise vermisst die schwarz-weiße Konzeption von Angebots- und Nachfrageseite nach Wohnraum an der ein oder anderen Stelle sicherlich graue Nuancierungen und mit dem Konzept des Policy Entrepreneurs wurde gezeigt, dass Individuen auch unabhängig von ihrer eigentlichen Funktion bestimmte Policies verfolgen oder behindern können. Die zunächst als homogen konzeptualisierten Akteursgruppen wurden also dort, wo die empirische Analyse es aufzeigte, auf einer Mikroebene der individuellen Akteure ausdifferenziert. 
Wohnungs- und sozialpolitische Implikationen

Die empirisch-analytisch geleitete Politikfeldanalyse nimmt eine "nüchterne Perspektive» ein und erklärt politische Prozesse ohne eigene Werturteile der Forscher*innen (Böcher/Töller 2019: 203). Dennoch liegen der Auswahl eines Forschungsvorhabens häufig normative Motive zugrunde: Inwieweit sollte Wohnraum für Menschen mit körperlichen und/oder geistigen Beeinträchtigungen dem Wohnungsmarkt überlassen bleiben oder politisch reguliert und unterstützt werden? Wie ließe sich eine solche politische Steuerung möglichst »sinnvoll« gestalten? Wie kann sie sich an den individuellen Problemlagen orientieren und zugleich mögliche Zielkonflikte berücksichtigen? Diese Arbeit hat gezeigt, dass die politischen Prozesse durch ihre Eigendynamik und die Interessen der beteiligten Akteure oft nicht (nur) an einer solchen Problemlösung orientiert sind.

So wäre es aus einer Problemlösungsorientierung heraus geboten, barrierefreien Wohnraum stärker auszudifferenzieren: Einige Bestandteile der Barrierefreiheit etwa wären ohne Weiteres in wohnungspolitische Logiken integrierbar, wohingegen andere Bestandteile im Widerstreit zum »einfachen« oder "günstigen« Bauen stehen. An dieser Stelle kann es helfen, Politikgestaltung als »Trial and Error«-Prozess zu begreifen, um praktisch bewährte Policy-Outputs zu identifizieren. Die 16 Bundesländer bieten hierfür eine dankbare Datenbasis, weshalb abschließend einige handlungsorientierte Implikationen diskutiert werden, die im Verlauf des Forschungsprozesses als »sinnvoll« im Sinne der politischen Problemlösung aufgetaucht sind.

Der untersuchte Policy-Mix reagiert auf unterschiedliche Teilbereiche des barrierefreien bzw. -reduzierten Wohnraums: Er adressiert sowohl den Neubau als auch den Bestandsumbau sowie unterschiedliche Zielgruppen von der Wohnungs- und Bauwirtschaft bis hin zu selbstnutzenden Eigentümer*innen. Diese breite Steuerungspalette darf nicht darüber hinwegtäuschen, dass einzig das Ordnungsrecht einen regulativen Rahmen setzt. Finanzielle und informationelle Angebote erreichen diejenigen, die im barrierefreien bzw. -reduzierten Wohnraum ohnehin eine Zielstellung sehen und darin unterstützt werden sollen. Eine flächendeckende, langfristige Herstellung von entsprechendem Wohnraum kann allerdings nur über verbindliche Vorgaben gewährleistet werden. Angesichts der demografischen Entwicklung und des bestehenden Wissens über barrierefreie Standards, die sich ohne größere Zielkonflikte in wohnungspolitische Logiken integrieren lassen, erscheint es geboten, nicht nur auf eine ökonomische oder informationelle Steuerung zu vertrauen. Dies gilt umso stärker, da mit dem heutigen Bau neuer Wohngebäude große Pfadabhängigkeiten erzeugt werden: Sie legen den Grundstein für den Wohnungsbestand der kommenden Jahrzehnte.

Direkt damit verbunden ist die Frage, was ein geeigneter Standard und eine geeignete Menge an entsprechend herzustellendem Wohnraum wäre. Das föderale Mehrebenensystem führt an dieser Stelle dazu, dass alle 16 Länder je für sich versuchen, Fragen der technischen Machbarkeit - zum Beispiel beim schwellenlosen Freisitz - zu klären. Auch wenn einige Bundesländer statistisch gesehen eine etwas »ältere« oder »kränkere« Bevölkerung haben und damit einen etwas größeren Bedarf an solchem Wohnraum haben als andere Bundesländer, ist es aus einer Problemlösungsperspektive heraus unverständlich, dass die entsprechenden Regelungen an den Landesgrenzen enden. Ei- 
ne für alle Länder verbindliche Musterbauordnung würde die Ressourcen bündeln, zu einer effizienteren Politikgestaltung beitragen und damit an fachlicher Expertise ausgerichtete Ergebnisse wahrscheinlicher machen. Sowohl die Angebotsseite als auch die Nachfrageseite nach Wohnraum fordern ein solches einheitliches Bauordnungsrecht ein. Aus einer pluralismuskritischen Perspektive muss hingegen berücksichtigt werden, dass sich auch bei Aushandlungsprozessen auf Bundesebene Akteure mit einer stärkeren Ressourcenausstattung besser durchsetzen könnten.

Im Abwägen der Sinnhaftigkeit einer bundesweiten Regelung sind außerdem die regional unterschiedlichen Wohnungsmärkte nicht außer Acht zu lassen. Insbesondere in der hessischen Fallstudie äußerten Interviewpartner*innen vermehrt den Wunsch nach einer differenzierten, an die lokalen Wohnraumbedarfe angepassten Wohnungspolitik. Solche kommunalen Regelungen können entweder als Alternative zu einem bundesweiten Basisstandard oder als Ergänzung dazu verstanden werden. Nordrhein-Westfalen etwa hat sich statt einer zunächst geplanten Quote für rollstuhlgerechten Wohnraum im Bauordnungsrecht für kommunale Zielvereinbarungen mit Städten wie Köln, Bonn oder Münster entschieden (MHKBG NRW 2020a). Hier bleibt abzuwarten, inwieweit diese seit 2019 bestehende Regelung eine bedarfsgerechte, kleinräumige Steuerung ermöglicht.

Eine von allen 16 Ländern als verbindlich anerkannte Musterbauordnung kann angesichts der föderalen, pfadabhängigen Aufgabenverteilung zudem leicht als nichtrealistische Instrumenten-Alternative abgetan werden. Verfechter*innen einer stärkeren Verankerung von Barrierefreiheit in der Wohnungspolitik können aber auch in einzelnen Bundesländern Inspiration dafür bekommen, was dort jeweils »technisch und finanziell machbar « sowie "politisch durchsetzbar« ist. So zeigt die soziale Wohnraumförderung für Bayern, Bremen und Nordrhein-Westfalen, dass flächendeckende barrierefreie Vorgaben durchaus mit der Zielvorgabe des bezahlbaren Wohnraums vereinbar sind. Zudem legen die differenzierten Regelungen im niedersächsischen und nordrhein-westfälischen Bauordnungsrecht nahe, dass weitreichende barrierefreie Standards in die breitere Wohnungspolitik integrierbar sind. Bei Einfamilienhäusern und Wohngebäuden mit wenigen Geschossen ist etwa die Sinnhaftigkeit eines Aufzugseinbaus zur absoluten Stufenlosigkeit sicherlich diskutabel. Hier bestehen klare Zielkonflikte zwischen der Forderung nach einer inklusiven Gesellschaft und ökonomischen Kostenabwägungen. Im mehrgeschossigen Wohnungsneubau indessen sind sozial- und wohnungspolitische Interessen durch eine fachlich fundierte Planung leichter miteinander in Einklang zu bringen, als es die politischen Prozesse bislang suggerieren. Dabei sollte der langfristige Nutzen einer inklusiven Wohnungspolitik auch in der Kalkulation eventueller Mehrkosten zum Herstellungszeitpunkt Berücksichtigung finden: Kostensteigerungen zum Status Quo können sich zukünftig vielfach ökonomisch und sozial auszahlen, etwa indem Umzüge in stationäre Pflegeeinrichtungen hinausgezögert oder Stürze und soziale Vereinsamung verhindert werden.

Insgesamt beschäftigen sich die Wohnungspolitik und damit auch die Wohnungspolitikforschung mit einem sogenannten »moving target « und müssen damit auf sich ständig wandelnde Wohnraumnachfragen reagieren. Veränderungen in der Lebensund Arbeitswelt, in der demografischen und sonstigen sozioökonomischen Struktur 
der Bevölkerung beeinflussen, wie, wo und mit wem Menschen heute und zukünftig leben möchten. Entsprechend verhält es sich auch beim barrierefreien bzw. -reduzierten Wohnraum. So gilt es beständig zu beobachten und bei Bedarf neu abzuwägen, was alles unter »baulicher Barrierefreiheit« zu verstehen ist. Das Kriterium der »Auffindbarkeit«, das über inklusionspolitische Gesetzgebungen sukzessive seinen Weg ins Bauordnungsrecht findet, weist auf die nun berücksichtigten Bedarfe von Menschen mit Sehbeeinträchtigungen hin (vgl. Kapitel 6.1.2). Psychische Erkrankungen wie dementielle oder autistische Leiden werden unter der baulichen Barrierefreiheit bislang allerdings vor allem in Fachkreisen außerhalb des politischen Raums diskutiert (vgl. bfb barrierefrei bauen 2020a; bfb barrierefrei bauen 2020b; Röhn 2012). Hier ist es wiederum entscheidend, wie erfolgreich sich entsprechende Spezialinteressen eine Stimme im politischen Prozess verschaffen können.

Ebenfalls ausgesprochen dynamisch ist die Entwicklung von verschiedenen mit dem Wohnen verbundenen Service-Dienstleistungen. Neben klassischen Trägereinrichtungen bieten immer mehr Wohnungsunternehmen sogenanntes betreutes Wohnen, Service-Wohnen oder Wohnen-Plus an (IP 33: 74; IP 39: 69ff.; IP 44: 20; IP 48: 68). Die bauliche Barrierefreiheit ist in diesem Kontext vielfach nur ein Baustein in einem breiteren Gesamtkonzept aus Pflege- und sonstigen Dienstleistungen sowie gemeinschaftlicher Alltagsgestaltung. Dabei verschwimmen die Grenzen zwischen ambulantem und stationärem Wohnen zusehends, womit sich entsprechende Angebote in einem Graubereich zwischen Bauordnungsrecht und Heimgesetzgebung befinden. Einerseits besteht hier ein politischer Handlungsbedarf zur rechtlichen Klärung, andererseits zeigt sich klar die Selbstregelungsfähigkeit des Marktes - der Wohnungsmarkt reagiert bereits von sich aus auf veränderte Wohnraumnachfragen, dann jedoch zu seinen Bedingungen und nach seinen wirtschaftlichen Logiken. Die politische Herausforderung besteht nun darin, die Balance zwischen staatlicher Intervention und wohnungswirtschaftlicher Aktivität so zu gestalten, dass die Interessen aller Marktteilnehmer*innen angemessen berücksichtigt werden: Sowohl die Interessen derjenigen, die Wohnraum zur Verfügung stellen, als auch derjenigen, die auf diesen Wohnraum angewiesen sind - dabei gilt es, die Schwächeren unter den letztgenannten besonders zu unterstützen. 